\title{
Existence of a Reversible T-Point Heteroclinic Cycle in a Piecewise Linear Version of the Michelson System*
}

\author{
Victoriano Carmona ${ }^{\dagger}$, Fernando Fernández-Sánchez ${ }^{\dagger}$, and Antonio E. Teruel ${ }^{\ddagger}$
}

\begin{abstract}
The proof of the existence of a global connection in differential systems is generally a difficult task. Some authors use numerical techniques to show this existence, even in the case of continuous piecewise linear systems. In this paper we give an analytical proof of the existence of a reversible T-point heteroclinic cycle in a continuous piecewise linear version of the widely studied Michelson system. The principal ideas of this proof can be extended to other piecewise linear systems.
\end{abstract}

Key words. piecewise linear systems, heteroclinic orbits, invariant manifolds

AMS subject classifications. 34C23, 34C37, 37G99

DOI. $10.1137 / 070709542$

1. Introduction. The existence of global connections in a differential system usually forces a complex dynamical behavior in a neighborhood of such connections. For instance, under the presence of a homoclinic cycle to a saddle-focus equilibrium point satisfying an eigenvalue ratio condition, the celebrated works of Shil'nikov $[26,27]$ ensure the existence of infinitely many periodic orbits of saddle type accumulating to the homoclinic cycle.

Moreover, the existence of a global connection in a differential system implies the appearance of subsidiary connections for certain perturbations of the system. For example, an analysis of the bifurcation structure of homoclinic cycles and subsidiary connections can be found in [15].

Heteroclinic cycles are also organizing centers of a very complex dynamic [10, 11, 12, 14$]$. In particular, Dumortier, Ibañez, and Kokubu [11] conjecture the existence of an infinite set of bifurcation phenomena, called a cocoon bifurcation [20], accumulating at a reversible T-point, that is, a point of the parameter space where a special kind of heteroclinic cycle satisfying some nondegeneracy condition appears. Furthermore, they explain the occurrence of such bifurcation phenomena as a consequence of the presence of this global connection.

Unfortunately, for nonlinear differential systems it is not easy to guarantee the existence of a global connection. Even though this is possible, some other extra conditions, for instance,

\footnotetext{
${ }^{*}$ Received by the editors November 29, 2007; accepted for publication (in revised form) by J. Meiss May 1, 2008; published electronically September 25, 2008.

http://www.siam.org/journals/siads/7-3/70954.html

${ }^{\dagger}$ Departamento de Matemática Aplicada II, Universidad de Sevilla, Escuela Superior de Ingenieros, Camino de los Descubrimientos s/n, 41092 Seville, Spain (vcarmona@us.es, fefesan@us.es). These authors were partially supported by the Ministerio de Ciencia y Tecnología, Plan Nacional I+D+I, under the projects MTM2004-04066, MTM200600847, and MTM2007-64193 and by the Conserjería de Educación y Ciencia de la Junta de Andalucía (TIC-0130, EXC/2005/FQM-872).

¥Departament de Matemàtiques i Informàtica, Universitat de les Illes Balears, Carretera de Valldemossa km. 7.5, 07122 Palma de Mallorca, Spain (antonioe.teruel@uib.es). This author was partially supported by the MCYT grant MTM2005-06098-C02-1 and by UIB grant UIB2005/6.
} 
the nondegeneracy conditions in the case of a reversible T-point heteroclinic cycle, cannot always be verified in a rigorous way. For example, the existence of a heteroclinic cycle in the Michelson system [25, 28], having an explicit expression for one of its heteroclinic orbits, is known $[16,18]$. Nevertheless, the genericity conditions that determine if such a heteroclinic cycle is a reversible T-point heteroclinic cycle remain to be verified [11].

On the other hand, there are a lot of papers devoted to the existence of global connections in piecewise linear differential systems $[2,8,9,22,23,24]$. Many of these works require numerical arguments to show the existence of the global connections. However, in [22], the authors provide an analytical proof of the existence of a homoclinic cycle in a three-dimensional piecewise linear system. In a similar way, in the present work we pay attention to the existence of a reversible T-point heteroclinic cycle in piecewise linear systems.

Now, some ideas for establishing the main result of the paper are introduced. The Michelson system is the one-parameter family of autonomous three-dimensional differential systems

$$
\left\{\begin{array}{l}
\dot{x}=y, \\
\dot{y}=z, \\
\dot{z}=d^{2}-y-\frac{1}{2} x^{2},
\end{array}\right.
$$

where the dot stands for the derivative with respect to $t$ and, without loss of generality, we can assume that $d \geq 0$. This family appears in the study of traveling wave solutions of the one-dimensional Kuramoto-Sivashinsky equation [25]. It also arises in the analysis of the unfolding of the nilpotent singularity of codimension three $[10,13]$.

For $d \neq 0$ the Michelson system has two equilibrium points, $\mathbf{p}_{ \pm}=( \pm d \sqrt{2}, 0,0)$, which are of saddle-focus type. The stable manifold $W^{s}\left(\mathbf{p}_{+}\right)$of $\mathbf{p}_{+}$(respectively, the unstable manifold $W^{u}\left(\mathbf{p}_{-}\right)$of $\left.\mathbf{p}_{-}\right)$is one-dimensional and the unstable manifold $W^{u}\left(\mathbf{p}_{+}\right)$of $\mathbf{p}_{+}$(respectively, the stable manifold $W^{s}\left(\mathbf{p}_{-}\right)$of $\left.\mathbf{p}_{-}\right)$is two-dimensional.

The vector field $\mathbf{f}$ associated to the Michelson system (1.1) satisfies the following important properties that affect the solution set:

- The divergence of $\mathbf{f}$ is identically zero. Therefore, the family is volume-preserving.

- The vector field $\mathbf{f}$ is invariant under the linear involution $\mathbf{R}(x, y, z)=(-x, y,-z)$ and sign reverse; that is,

$$
\mathbf{R}(\mathbf{f}(\mathbf{x}))=-\mathbf{f}(\mathbf{R}(\mathbf{x}))
$$

where $\mathbf{x}=(x, y, z)^{T}$.

From the second property, the following dynamical consequences are obtained:

- Let $\phi(t ; \mathbf{p})$ denote the flow of system (1.1). The equality $\phi(t ; \mathbf{R}(\mathbf{p}))=\mathbf{R}(\phi(-t ; \mathbf{p}))$ holds, and we say that the family is time-reversible with respect to the linear involution $\mathbf{R}$.

- If $\mathbf{p}$ is a point on the $y$-axis, then $\mathbf{R}(\mathbf{p})=\mathbf{p}$. Therefore, the orbit through $\mathbf{p}$ is reversible with respect to $\mathbf{R}$, that is, $\phi(t ; \mathbf{p})=\mathbf{R}(\phi(-t ; \mathbf{p}))$, and the $y$-axis is called the reversibility axis.

- By the reversibility, the stable and unstable manifolds of $\mathbf{p}_{-}$satisfy $W^{s}\left(\mathbf{p}_{-}\right)=$ $\mathbf{R}\left(W^{u}\left(\mathbf{p}_{+}\right)\right)$and $W^{u}\left(\mathbf{p}_{-}\right)=\mathbf{R}\left(W^{s}\left(\mathbf{p}_{+}\right)\right)$, respectively.

An interesting object of the dynamics of the Michelson system which has been widely studied (see $[11,16,19,20]$ and the references therein) is the so-called reversible T-point 
heteroclinic cycle. Following [11], we say that the Michelson system (1.1) has a reversible Tpoint heteroclinic cycle $\Gamma=\left\{\mathbf{p}_{+}\right\} \cup \rho_{ \pm} \cup\left\{\mathbf{p}_{-}\right\} \cup \rho_{\mp}$ for the parameter value $d_{0}$ if the following hold:

(a) The two-dimensional manifolds $W^{u}\left(\mathbf{p}_{+}\right)$and $W^{s}\left(\mathbf{p}_{-}\right)$have a transversal intersection along the heteroclinic orbit $\rho_{ \pm}$.

(b) As the parameter $d$ is varied around $d_{0}$ the heteroclinic orbit $\rho_{\mp}$ unfolds generically. That is, the one-dimensional manifolds $W^{u}\left(\mathbf{p}_{-}\right)$and $W^{s}\left(\mathbf{p}_{+}\right)$intersect at $\rho_{\mp}$ for $d=d_{0}$ and the "distance" between $W^{u}\left(\mathbf{p}_{-}\right)$and $W^{s}\left(\mathbf{p}_{+}\right)$measured in a transverse plane is diffeomorphic to $\mu=d-d_{0}$.

The value $d_{0}$ is usually called a reversible T-point. Note that some authors [11] use the name Bykov cycle to refer to a T-point heteroclinic cycle $[3,4,5]$.

By abuse of notation, we can call the orbit $\rho_{\mp}$ a one-dimensional heteroclinic orbit, because this orbit corresponds to the one-dimensional invariant manifolds of the singular points, and the orbit $\rho_{ \pm}$a two-dimensional heteroclinic orbit because it is contained in the intersection set of the two-dimensional invariant manifolds of the singular points.

From several recent works it is possible to discern that piecewise linear systems are able to reproduce the dynamics of differentiable systems. Thus, it is natural to wonder if a suitable continuous piecewise linear version of the Michelson system with a reversible T-point exists.

An easy way to obtain a continuous piecewise linear system from the Michelson system is to perform, for $d \neq 0$, the change of variables $x \rightarrow x / d^{2}, y \rightarrow y / d^{2}, z \rightarrow z / d^{2}$ followed by the change of function $x^{2} \rightarrow|x|$. This procedure transforms system (1.1) into

$$
\left\{\begin{array}{l}
\dot{x}=y \\
\dot{y}=z \\
\dot{z}=1-y-c|x|
\end{array}\right.
$$

where $c=\frac{d^{2}}{2}$. Note that this system is also volume-preserving and time-reversible with respect to the involution $\mathbf{R}$.

Due to the lack of differentiability of the piecewise linear vector fields, the generic tools of the analysis of differentiable systems cannot be applied. Therefore, the techniques used in [11] for the Michelson system are useless for our piecewise linear continuous version. Nevertheless, we also show that some dynamical aspects of the Michelson system remain in our piecewise linear version.

System (1.2) is formed by two linear systems separated by the plane $\{x=0\}$, called the separation plane, and it can be written in a matricial form as

$$
\dot{\mathbf{x}}= \begin{cases}A^{+} \mathbf{x}+\mathbf{e}_{3} & \text { if } x \geq 0 \\ A^{-} \mathbf{x}+\mathbf{e}_{3} & \text { if } x \leq 0\end{cases}
$$

with

$$
A^{+}=\left(\begin{array}{ccc}
0 & 1 & 0 \\
0 & 0 & 1 \\
-c & -1 & 0
\end{array}\right), A^{-}=\left(\begin{array}{ccc}
0 & 1 & 0 \\
0 & 0 & 1 \\
c & -1 & 0
\end{array}\right), \text { and } \mathbf{e}_{3}=\left(\begin{array}{c}
0 \\
0 \\
1
\end{array}\right)
$$

In the half-space $\{x<0\}$, the system has exactly one equilibrium point $\mathbf{p}_{-}=(-1 / c, 0,0)^{T}$ which is a saddle-focus point. Let $\lambda>0$ and $\alpha \pm \mathrm{i} \beta$ be the eigenvalues of the Jacobian matrix 
at $\mathbf{p}_{-}$. This clearly implies that

$$
c=\lambda\left(1+\lambda^{2}\right), \quad \alpha=-\frac{\lambda}{2}, \quad \beta=\frac{\sqrt{4+3 \lambda^{2}}}{2} .
$$

By the reversibility with respect to $\mathbf{R}$, there exists exactly one saddle-focus equilibrium $\mathbf{p}_{+}=$ $(1 / c, 0,0)^{T}$ in the half-space $\{x>0\}$ whose eigenvalues are given by $-\lambda$ and $-\alpha \pm \mathrm{i} \beta$.

Using the expression of the parameter $c$ given in (1.4), system (1.2) can be written as

$$
\left\{\begin{array}{l}
\dot{x}=y, \\
\dot{y}=z, \\
\dot{z}=1-y-\lambda\left(1+\lambda^{2}\right)|x|,
\end{array}\right.
$$

and the parameter $\lambda>0$ can be chosen as the fundamental parameter of the family.

In the particular case of piecewise linear systems, global connections can be classified attending to the number of intersections with the separation plane. Using the notation introduced above, we say that a reversible T-point heteroclinic cycle $\Gamma$ of system $(1.5)$ is $(n, m)$ if the one-dimensional heteroclinic orbit $\rho_{\mp}$ intersects the separation plane $\{x=0\}$ at exactly $n$ points and the two-dimensional heteroclinic orbit $\rho_{ \pm}$intersects $\{x=0\}$ at exactly $m$ points.

Obviously, the reversibility of system (1.5) forces $n$ to be odd. As we will see later, due to the local linear shape of the one-dimensional invariant manifolds, $n$ has to be different than one. So, the $(3,1)$ reversible T-point heteroclinic cycle can be considered to be the simplest one and its existence will be the main goal of this work, as it is summarized in the following theorem.

Theorem 1.1. There exists a value $\lambda_{1} \in(1 / 2,1)$ such that the piecewise linear version $(1.5)$ of the Michelson system has a $(3,1)$ reversible T-point heteroclinic cycle $\Gamma$ for $\lambda=\lambda_{1}$.

Some numerical computations allow us to obtain $\lambda_{1} \approx 0.65153556$. In fact, its boundary values $1 / 2$ and 1 do not have any dynamical meaning, and they have been chosen for the sake of simplicity of the handmade calculations involved in the proof.

Piecewise linear system (1.5) has been obtained from the Michelson system by using a natural transformation. This transformation preserves not only the look of the equations but also the properties of reversibility and volume-preservation. We must emphasize that this is not casual. In fact, considering a piecewise linear continuous system with separation plane $\{x=0\}$ and two saddle-focus equilibria, and assuming the reversibility, volume-preservation, and nondegeneracy conditions, the unique system that can be obtained is (1.5) except for linear changes of variables and time. More precisely, a piecewise linear continuous system with separation plane $\{x=0\}$ under the hypothesis of observability can be written as

$$
\left\{\begin{array}{l}
\dot{x}=t^{ \pm} x-y, \\
\dot{y}=m^{ \pm} x-z, \\
\dot{z}=d^{ \pm} x-1
\end{array}\right.
$$

where parameters $t^{+}, m^{+}$, and $d^{+}$correspond to the linear system in the half-space $\{x>0\}$ while $t^{-}, m^{-}$, and $d^{-}$correspond to the linear system in the half-space $\{x<0\}$. Observability is a nondegeneracy condition, which means that the dynamics of the system cannot be uncoupled $[6,7]$. The reversibility condition with respect to $\mathbf{R}$ implies that $t^{-}=-t^{+}$, 
$m^{-}=m^{+}$, and $d^{-}=-d^{+}$. On the other hand, system (1.6) is volume-preserving if and only if $t^{+}=t^{-}=0$. Now, the existence of two saddle-focus equilibria forces the parameters $d^{+}$ and $m^{+}$to be positive. Hence, system (1.6) is really

$$
\left\{\begin{array}{l}
\dot{x}=-y, \\
\dot{y}=m x-z, \\
\dot{z}=d|x|-1,
\end{array}\right.
$$

where $d=d^{+}>0$ and $m=m^{+}>0$. The trivial linear change of variables and time,

$$
X=-m^{3 / 2} x, \quad Y=m y, \quad Z=m^{3 / 2} x-m^{1 / 2} z, \quad \tau=m^{1 / 2} t,
$$

transforms the system into

$$
\left\{\begin{array}{l}
X^{\prime}=Y, \\
Y^{\prime}=Z, \\
Z^{\prime}=1-Y-\frac{d}{m^{3 / 2}}|X|,
\end{array}\right.
$$

where the prime stands for the derivative with respect to $\tau$. This is the piecewise linear version of the Michelson system previously obtained.

The rest of the paper is devoted to the proof of Theorem 1.1, and it is organized as follows. In section 2 we describe the basic geometric elements of the problem. In section 3 we prove the existence, for every $\lambda$ in a semi-infinite interval, of a two-dimensional heteroclinic orbit with exactly one intersection point with the plane $\{x=0\}$. For one of these values of $\lambda$, there exists a one-dimensional heteroclinic orbit with exactly three intersection points with $\{x=0\}$, as is proved in section 4 . From this follows the existence of a simple heteroclinic cycle $\Gamma$. In section 5 we prove that $\Gamma$ satisfies the nondegeneracy conditions of a reversible T-point heteroclinic cycle.

2. Some geometric elements of the flow. In this section we describe the behavior of the flow crossing the plane $\{x=0\}$ and the basic elements of the linear dynamics locally contained in the half-spaces $\{x<0\}$ and $\{x>0\}$.

For every point $\mathbf{p}=\left(x_{\mathbf{p}}, y_{\mathbf{p}}, z_{\mathbf{p}}\right)^{T} \in \mathbb{R}^{3}$ we denote by $\mathbf{x}_{\mathbf{p}}(t ; \lambda)=\left(x_{\mathbf{p}}(t ; \lambda), y_{\mathbf{p}}(t ; \lambda)\right.$, $\left.z_{\mathbf{p}}(t ; \lambda)\right)^{T}$ the solution of the system $(1.5)$ with parameter $\lambda$ and initial condition $\mathbf{x}_{\mathbf{p}}(0 ; \lambda)=\mathbf{p}$. The corresponding orbit is denoted by $\gamma_{\mathbf{p}}$.

If $x_{\mathbf{p}}=0$ and $y_{\mathbf{p}}>0$, then the orbit $\gamma_{\mathbf{p}}$ crosses transversally the plane $\{x=0\}$ with $x_{\mathbf{p}}(-t ; \lambda)<0$ and $x_{\mathbf{p}}(t ; \lambda)>0$ for $t>0$ small enough. If $x_{\mathbf{p}}(t ; \lambda)$ vanishes in $(0,+\infty)$, then we define the flying time $t_{\mathbf{p}}^{+}$as the positive value such that $x_{\mathbf{p}}\left(t_{\mathbf{p}}^{+} ; \lambda\right)=0$ and $x_{\mathbf{p}}(t ; \lambda)>0$ in $\left(0, t_{\mathbf{p}}^{+}\right)$. In such a case, we define the Poincaré map $\Pi_{+}$at the point $\mathbf{p}$ as $\Pi_{+}(\mathbf{p})=$ $\left(0, y_{\mathbf{p}}\left(t_{\mathbf{p}}^{+} ; \lambda\right), z_{\mathbf{p}}\left(t_{\mathbf{p}}^{+} ; \lambda\right)\right)^{T}$. Note that the Poincaré map $\Pi_{+}$depends only on the linear system $\dot{\mathbf{x}}=A^{+} \mathbf{x}+\mathbf{e}_{3}$ given in (1.3).

If $x_{\mathbf{p}}=0$ and $y_{\mathbf{p}}<0$, then the orbit $\gamma_{\mathbf{p}}$ crosses transversally the plane $\{x=0\}$ with $x_{\mathbf{p}}(-t ; \lambda)>0$ and $x_{\mathbf{p}}(t ; \lambda)<0$ for $t>0$ small enough. If $x_{\mathbf{p}}(t ; \lambda)$ vanish in $(0,+\infty)$, then we define the flying time $t_{\mathbf{p}}^{-}$as the positive value such that $x_{\mathbf{p}}\left(t_{\mathbf{p}}^{-} ; \lambda\right)=0$ and $x_{\mathbf{p}}(t ; \lambda)<0$ in $\left(0, t_{\mathbf{p}}^{-}\right)$. In such a case, we define the Poincaré map $\Pi_{-}$at the point $\mathbf{p}$ as $\Pi_{-}(\mathbf{p})=$ $\left(0, y_{\mathbf{p}}\left(t_{\mathbf{p}}^{-} ; \lambda\right), z_{\mathbf{p}}\left(t_{\mathbf{p}}^{-} ; \lambda\right)\right)^{T}$. This map depends only on the linear system $\dot{\mathbf{x}}=A^{-} \mathbf{x}+\mathbf{e}_{3}$. 


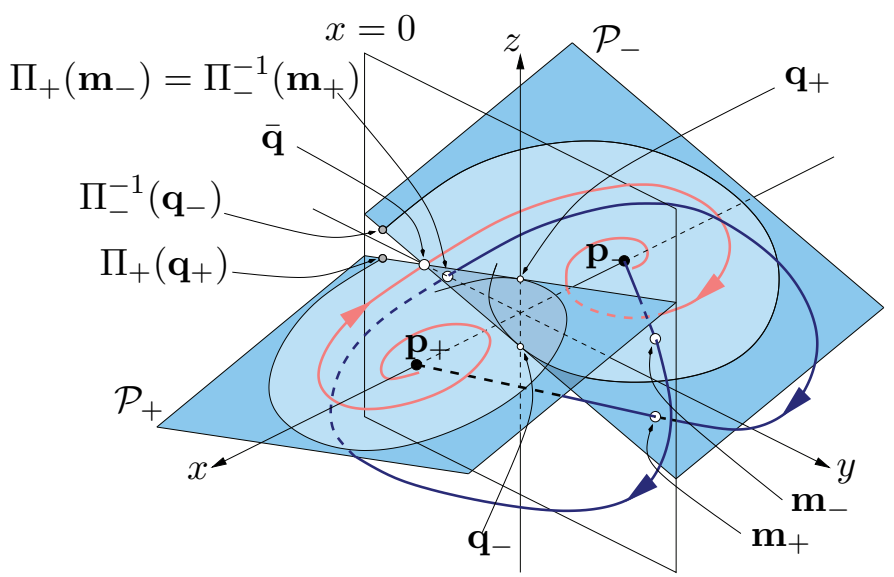

Figure 1. Some geometric elements of the flow.

If $\mathbf{p}$ belongs to the $z$-axis, i.e., $x_{\mathbf{p}}=0$ and $y_{\mathbf{p}}=0$, then $\mathbf{p}$ is called a contact point of the flow of system (1.5) with the plane $\{x=0\}$ because the vector field at this point is tangent to the plane. Following [21], the first coordinate of the Taylor expansion of $\mathbf{x}_{\mathbf{p}}(t ; \lambda)-\mathbf{p}$ at $t=0$ is

$$
\mathbf{e}_{1}^{T}\left(\mathbf{x}_{\mathbf{p}}(t ; \lambda)-\mathbf{p}\right)=z_{\mathbf{p}} \frac{t^{2}}{2}+\frac{t^{3}}{3 !}+\mathbf{e}_{1}^{T} \mathbf{x}_{\mathbf{p}}^{(4)}(\xi ; \lambda) \frac{t^{4}}{4 !} .
$$

Hence, if $z_{\mathbf{p}}<0$, then orbit $\gamma_{\mathbf{p}}$ is locally contained in the half-space $\{x \leq 0\}$; if $z_{\mathbf{p}}>0$, then $\gamma_{\mathbf{p}}$ is locally contained in the half-space $\{x \geq 0\}$; and if $z_{\mathbf{p}}=0$, then $\gamma_{\mathbf{p}}$ crosses the plane $\{x=0\}$ from the half-space $\{x<0\}$ to the half-space $\{x>0\}$.

Now we describe the basic elements of the linear dynamics in every half-space; all this information is summarized in Figure 1.

The stable manifold $W^{s}\left(\mathbf{p}_{+}\right)$of $\mathbf{p}_{+}$contains the half-line $\mathcal{L}_{+}=\left\{\mathbf{p}_{+}+\mu\left(1,-\lambda, \lambda^{2}\right)\right.$ : $\left.-\frac{1}{\lambda\left(1+\lambda^{2}\right)} \leq \mu<\infty\right\}$ generated by the eigenvector associated to the eigenvalue $-\lambda$ of the matrix $A^{+}$. The half-line $\mathcal{L}_{+}$and the plane $\{x=0\}$ intersect at the point

$$
\mathbf{m}_{+}=\left(0, \frac{1}{1+\lambda^{2}},-\frac{\lambda}{1+\lambda^{2}}\right)^{T} .
$$

The unstable two-dimensional manifold $W^{u}\left(\mathbf{p}_{+}\right)$is locally contained in the half-plane

$$
\mathcal{P}_{+}=\left\{\lambda\left(1+\lambda^{2}\right) x-\lambda^{2} y+\lambda z=1: x \geq 0\right\},
$$

which is called the focal half-plane of $\mathbf{p}_{+}$. This half-plane is obtained from the eigenvectors associated to the complex eigenvalues of $A^{+}$. The half-plane $\mathcal{P}_{+}$and the separation plane $\{x=0\}$ intersect along the straight line

$$
\mathcal{D}_{+}=\left\{-\lambda^{2} y+\lambda z=1\right\} .
$$

Let us emphasize that not every point in $\mathcal{D}_{+}$belongs to the unstable manifold $W^{u}\left(\mathbf{p}_{+}\right)$; see Figure 1. The intersection point of $\mathcal{D}_{+}$and the $z$-axis is $\mathbf{q}_{+}=\left(0,0, \frac{1}{\lambda}\right)^{T}$. Since $\mathbf{q}_{+}$is a contact 
point, the orbit $\gamma_{\mathbf{q}_{+}}$is tangent to the separation plane $\{x=0\}$ at $\mathbf{q}_{+}$. Thus, the segment $\mathcal{S}_{+} \subset \mathcal{D}_{+}$with endpoints $\mathbf{q}_{+}$and $\Pi_{+}\left(\mathbf{q}_{+}\right)$is contained in $W^{u}\left(\mathbf{p}_{+}\right)$.

The unstable manifold $W^{u}\left(\mathbf{p}_{-}\right)$of $\mathbf{p}_{-}$contains the half-line $\mathcal{L}_{-}=\left\{\mathbf{p}_{-}-\mu\left(1, \lambda, \lambda^{2}\right)\right.$ : $\left.-\frac{1}{\lambda\left(1+\lambda^{2}\right)} \leq \mu<\infty\right\}$ generated by the eigenvector associated to the eigenvalue $\lambda$ of the matrix $A^{-}$. The half-line and the plane $\{x=0\}$ intersect at the point

$$
\mathbf{m}_{-}=\left(0, \frac{1}{1+\lambda^{2}}, \frac{\lambda}{1+\lambda^{2}}\right)^{T}
$$

The stable two-dimensional manifold $W^{s}\left(\mathbf{p}_{-}\right)$is locally contained in the half plane

$$
\mathcal{P}_{-}=\left\{\lambda\left(1+\lambda^{2}\right) x+\lambda^{2} y+\lambda z=-1: x \leq 0\right\},
$$

which is called the focal half-plane of $\mathbf{p}_{-}$. This half-plane is obtained from the eigenvectors associated to the complex eigenvalues of $A^{-}$. The half-plane $\mathcal{P}_{-}$and the separation plane $\{x=0\}$ intersect along the straight line

$$
\mathcal{D}_{-}=\left\{\lambda^{2} y+\lambda z=-1\right\}
$$

Let us emphasize that not every point in $\mathcal{D}_{-}$belongs to the stable manifold $W^{s}\left(\mathbf{p}_{-}\right)$. The intersection point of $\mathcal{D}_{-}$and the $z$-axis is $\mathbf{q}_{-}=\left(0,0,-\frac{1}{\lambda}\right)^{T}$. Since $\mathbf{q}_{-}$is a contact point, the orbit $\gamma_{\mathbf{q}_{-}}$is tangent to the separation plane $\{x=0\}$ at $\mathbf{q}_{-}$. Thus, the segment $\mathcal{S}_{-} \subset \mathcal{D}_{-}$with endpoints $\mathbf{q}_{-}$and $\Pi_{-}^{-1}\left(\mathbf{q}_{-}\right)$is contained in $W^{s}\left(\mathbf{p}_{-}\right)$.

3. Existence of a two-dimensional heteroclinic orbit. In this section we prove the existence of a simple two-dimensional heteroclinic orbit $\rho_{ \pm}$, that is, a heteroclinic orbit $\rho_{ \pm} \subset$ $W^{u}\left(\mathbf{p}_{+}\right) \cap W^{s}\left(\mathbf{p}_{-}\right)$which intersects the plane $\{x=0\}$ at exactly one point $\overline{\mathbf{q}}$.

A necessary and sufficient condition for the existence of the orbit $\rho_{ \pm}$is $\overline{\mathbf{q}} \in \mathcal{S}_{-} \cap \mathcal{S}_{+}$. This implies that $\overline{\mathbf{q}}=\left(0,-\lambda^{-2}, 0\right)^{T}$, because it is the intersection point of the straight lines $\mathcal{D}_{+}$ and $\mathcal{D}_{-}$. We now proceed to look for the values of the parameter $\lambda$ for which the point $\overline{\mathbf{q}}$ belongs to $\mathcal{S}_{-}$and, by reversibility, to $\mathcal{S}_{+}$.

By definition, the segment $\mathcal{S}_{-}$is defined by the endpoints $\mathbf{q}_{-}$and $\Pi_{-}^{-1}\left(\mathbf{q}_{-}\right)$. Since the third coordinate of $\mathbf{q}_{-}$is negative and $\overline{\mathbf{q}}$ has the third coordinate equal to zero, then $\overline{\mathbf{q}} \in \mathcal{S}_{-}$ if and only if the third coordinate of $\Pi_{-}^{-1}\left(\mathbf{q}_{-}\right)$is nonnegative. Therefore, system (1.5) has a simple two-dimensional heteroclinic orbit $\rho_{ \pm}$if and only if there exist $t_{0}>0$ and $\lambda_{0}>0$ such that $(t ; \lambda)=\left(t_{0} ; \lambda_{0}\right)$ is a solution of the system

$$
\left\{\begin{array}{l}
x_{\mathbf{q}_{-}}(-t ; \lambda)=0 \\
z_{\mathbf{q}_{-}}(-t ; \lambda) \geq 0
\end{array}\right.
$$

with

$$
x_{\mathbf{q}_{-}}\left(-t ; \lambda_{0}\right)<0 \text { for every } t \in\left(0, t_{0}\right) .
$$

We emphasize that condition (3.2) ensures that the point $\mathbf{x}_{\mathbf{q}_{-}}\left(-t_{0} ; \lambda_{0}\right)$ is the preimage of $\mathbf{q}_{-}$ by the Poincaré map $\Pi_{-}$. 
Taking into account condition (3.2), the expressions of $x_{\mathbf{q}_{-}}$and $z_{\mathbf{q}_{-}}$in (3.1) can be obtained by integrating the linear system in the half-space $\{x<0\}$ in backward time with initial condition $\mathbf{x}(0 ; \lambda)=\mathbf{q}_{-}$. Thus, system (3.1) can be written as

$$
\left\{\begin{array}{l}
x_{\mathbf{q}_{-}}(-t ; \lambda)=-\frac{1}{\lambda\left(1+\lambda^{2}\right)}\left[1-e^{\frac{\lambda}{2} t}\left(\cos \left(\frac{\sqrt{4+3 \lambda^{2}}}{2} t\right)-\frac{\lambda}{\sqrt{4+3 \lambda^{2}}} \sin \left(\frac{\sqrt{4+3 \lambda^{2}}}{2} t\right)\right)\right]=0, \\
z_{\mathbf{q}_{-}}(-t ; \lambda)=-\frac{1}{\lambda} e^{\frac{\lambda}{2} t}\left[\cos \left(\frac{\sqrt{4+3 \lambda^{2}}}{2} t\right)+\frac{\lambda}{\sqrt{4+3 \lambda^{2}}} \sin \left(\frac{\sqrt{4+3 \lambda^{2}}}{2} t\right)\right] \geq 0 .
\end{array}\right.
$$

Using the function $\varphi(\tau, \gamma)=1-e^{\gamma \tau}(\cos (\tau)-\gamma \sin (\tau))$ defined in [1], system (3.3) can be rewritten as

$$
\left\{\begin{array}{l}
-\frac{1}{\lambda\left(1+\lambda^{2}\right)} \varphi\left(-\frac{\sqrt{4+3 \lambda^{2}}}{2} t,-\frac{\lambda}{\sqrt{4+3 \lambda^{2}}}\right)=0 \\
-\frac{1}{\lambda} e^{\frac{\lambda}{2} t}\left(1-\varphi\left(\frac{\sqrt{4+3 \lambda^{2}}}{2} t,-\frac{\lambda}{\sqrt{4+3 \lambda^{2}}}\right)\right) \geq 0
\end{array}\right.
$$

Hence, the existence of a solution $(t ; \lambda)=\left(t_{0} ; \lambda_{0}\right)$ of (3.1) satisfying inequality (3.2) is equivalent to the existence of a solution $(t ; \lambda)=\left(t_{0} ; \lambda_{0}\right)$ of system

$$
\left\{\begin{array}{l}
\varphi\left(-\frac{\sqrt{4+3 \lambda^{2}}}{2} t,-\frac{\lambda}{\sqrt{4+3 \lambda^{2}}}\right)=0 \\
\varphi\left(\frac{\sqrt{4+3 \lambda^{2}}}{2} t,-\frac{\lambda}{\sqrt{4+3 \lambda^{2}}}\right) \geq 1
\end{array}\right.
$$

with $t_{0}>0, \lambda_{0}>0$ and such that

$$
\varphi\left(-\frac{\sqrt{4+3 \lambda_{0}^{2}}}{2} t,-\frac{\lambda_{0}}{\sqrt{4+3 \lambda_{0}^{2}}}\right)>0
$$

for every $t$ in $\left(0, t_{0}\right)$.

In the next result, whose proof is direct, we compile elementary properties of function $\varphi(\tau, \gamma)$, some of which can be found in [1].

Lemma 3.1. Function $\varphi(\tau, \gamma)=1-e^{\gamma \tau}(\cos (\tau)-\gamma \sin (\tau))$ satisfies the following properties.

(i) $\varphi(-\tau,-\gamma)=\varphi(\tau, \gamma)$.

(ii) $\frac{\partial \varphi}{\partial \tau}=\left(1+\gamma^{2}\right) e^{\gamma \tau} \sin (\tau)$ and $\frac{\partial^{2} \varphi}{\partial \tau^{2}}=\left(1+\gamma^{2}\right) e^{\gamma \tau}(\cos (\tau)+\gamma \sin (\tau))$.

(iii) For every fixed $\gamma>0$ the function $\varphi(\tau, \gamma)$ reaches its local maxima and minima values, respectively, at $\tau_{2 k+1}=(2 k+1) \pi$ and $\tau_{2 k}=2 k \pi$ with $k \in \mathbb{Z}$. Moreover, $\varphi\left(\tau_{2 k+1}, \gamma\right)=$ $1+e^{\gamma \tau_{2 k+1}}$ and $\varphi\left(\tau_{2 k}, \gamma\right)=1-e^{\gamma \tau_{2 k}}$.

(iv) There exists a unique function $\hat{\tau}_{1}:(0,+\infty) \rightarrow(\pi, 2 \pi)$ such that $\varphi\left(\hat{\tau}_{1}(\gamma), \gamma\right)=0$, $\varphi(\tau, \gamma)>0$ for $\tau \in\left(0, \hat{\tau}_{1}(\gamma)\right)$ and $\varphi(\tau, \gamma)<0$ for $\tau \in\left(\hat{\tau}_{1}(\gamma), 2 \pi\right)$.

(v) $\frac{\partial \varphi}{\partial \gamma}=e^{\gamma \tau}(-\tau \cos (\tau)+(1+\gamma \tau) \sin (\tau))$.

(vi) There exists a unique function $\hat{\tau}_{2}:(0,+\infty) \rightarrow(-2 \pi,-\pi)$ such that $\varphi\left(\hat{\tau}_{2}(\gamma), \gamma\right)=1$ and $\varphi(\tau, \gamma)>1$ for $\tau \in\left(\hat{\tau}_{2}(\gamma),-\pi\right)$.

The shape of functions $\hat{\tau}_{1}$ and $\hat{\tau}_{2}$ introduced in the previous lemma are described in the next result. Some of these properties are shown in Figure 2.

Lemma 3.2. The following properties of functions $\hat{\tau}_{1}$ and $\hat{\tau}_{2}$, defined in Lemma 3.1, hold.

(i) The function $\hat{\tau}_{1}$ is differentiable, $\frac{d \hat{\tau}_{1}}{d \gamma}<0, \lim _{\gamma \backslash 0} \hat{\tau}_{1}(\gamma)=2 \pi$, and $\lim _{\gamma / \infty} \hat{\tau}_{1}(\gamma)=\pi$. 


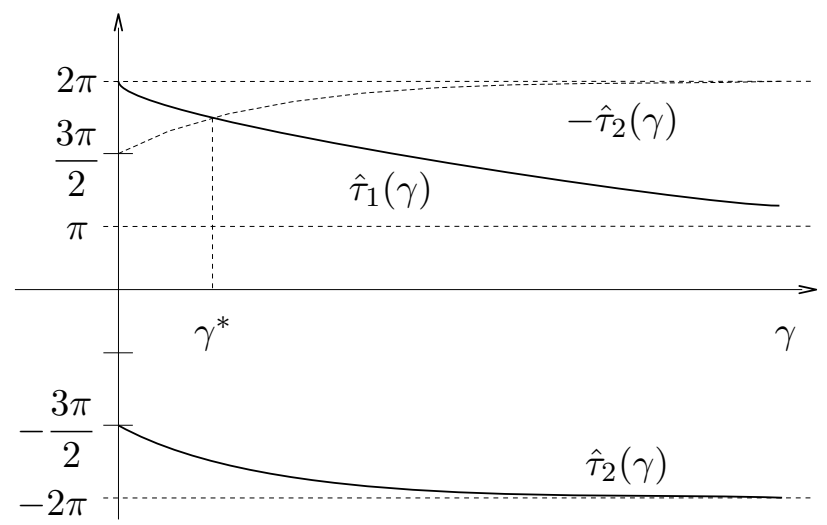

Figure 2. Qualitative behavior of functions $\hat{\tau}_{1}$ and $\hat{\tau}_{2}$. $-2 \pi$

(ii) The function $\hat{\tau}_{2}$ is differentiable, $\frac{d \hat{\tau}_{2}}{d \gamma}<0, \lim _{\gamma \backslash 0} \hat{\tau}_{2}(\gamma)=-\frac{3 \pi}{2}$, and $\lim _{\gamma} \nearrow_{\infty} \hat{\tau}_{2}(\gamma)=$

(iii) There exists a unique value $\gamma^{*}>0$ such that $-\hat{\tau}_{2}\left(\gamma^{*}\right)=\hat{\tau}_{1}\left(\gamma^{*}\right)$. This value satisfies $\gamma^{*}<\frac{1}{\sqrt{19}}$. Moreover, $-\hat{\tau}_{2}(\gamma)<\hat{\tau}_{1}(\gamma)$ in $\left(0, \gamma^{*}\right)$ and $-\hat{\tau}_{2}(\gamma)>\hat{\tau}_{1}(\gamma)$ in $\left(\gamma^{*},+\infty\right)$.

(iv) If $0<\gamma<\gamma^{*}$, then $\varphi\left(-\hat{\tau}_{1}(\gamma), \gamma\right)<1$, and $\varphi\left(-\hat{\tau}_{1}(\gamma), \gamma\right)>1$ if $\gamma>\gamma^{*}$.

Proof. (i) Since $\hat{\tau}_{1}(\gamma) \in(\pi, 2 \pi)$, by the implicit function theorem it follows that $\hat{\tau}_{1}$ is a differentiable function and

$$
\left.\frac{d \hat{\tau}_{1}}{d \gamma}\right|_{\gamma}=\frac{\hat{\tau}_{1}(\gamma) e^{-\gamma \hat{\tau}_{1}(\gamma)}-\sin \left(\hat{\tau}_{1}(\gamma)\right)}{\left(1+\gamma^{2}\right) \sin \left(\hat{\tau}_{1}(\gamma)\right)}<0
$$

Taking the limit as $\gamma$ tends to zero in the implicit expression $\varphi\left(\hat{\tau}_{1}(\gamma), \gamma\right)=0$, it is easy to check that $\lim _{\gamma \backslash 0} \cos \left(\hat{\tau}_{1}(\gamma)\right)=1$ and $\lim _{\gamma / \infty} \sin \left(\hat{\tau}_{1}(\gamma)\right)=0$. Hence, $\lim _{\gamma \backslash 0} \hat{\tau}_{1}(\gamma)=2 \pi$ and $\lim _{\gamma / \infty} \hat{\tau}_{1}(\gamma)=\pi$.

(ii) This statement follows by the same arguments as in the previous item.

(iii) By the definition of $\varphi$ and $\hat{\tau}_{2}$ it follows that, for every $\gamma>0, \cos \left(\hat{\tau}_{2}(\gamma)\right)=\gamma \sin \left(\hat{\tau}_{2}(\gamma)\right)$ and $\hat{\tau}_{2}(\gamma) \in\left(-2 \pi,-\frac{3 \pi}{2}\right)$. Fixing $\gamma=\frac{1}{\sqrt{19}}$ it is easy to obtain

$$
\cos \left(\hat{\tau}_{2}\left(\frac{1}{\sqrt{19}}\right)\right)=\frac{1}{2 \sqrt{5}} \text { and } \sin \left(\hat{\tau}_{2}\left(\frac{1}{\sqrt{19}}\right)\right)=\frac{\sqrt{19}}{2 \sqrt{5}}
$$

This clearly gives $\varphi\left(-\hat{\tau}_{2}\left(\frac{1}{\sqrt{19}}\right), \frac{1}{\sqrt{19}}\right)=1-\frac{1}{\sqrt{5}} e^{-\frac{\hat{\tau}_{2}\left(\frac{1}{\sqrt{19}}\right)}{\sqrt{19}}}$, which is negative because of $\hat{\tau}_{2}(\gamma)<$ $-\frac{3 \pi}{2}$.

On the other hand, since $\varphi\left(\hat{\tau}_{1}\left(\frac{1}{\sqrt{19}}\right), \frac{1}{\sqrt{19}}\right)=0$ and $\varphi\left(\tau, \frac{1}{\sqrt{19}}\right)$ is a monotone decreasing function for $\tau \in(\pi, 2 \pi)$ (see Lemma 3.1(iii)), then $\hat{\tau}_{1}\left(\frac{1}{\sqrt{19}}\right)<-\hat{\tau}_{2}\left(\frac{1}{\sqrt{19}}\right)$.

Following statements (i) and (ii) in this lemma, the auxiliary function $h(\gamma)=\hat{\tau}_{1}(\gamma)+\hat{\tau}_{2}(\gamma)$ satisfies that $h^{\prime}(\gamma)<0$ for every $\gamma>0$ and $\lim _{\gamma \backslash 0} h(\gamma)=\frac{\pi}{2}>0$. Since $h\left(\frac{1}{\sqrt{19}}\right)<0$, there 
exists a value $\gamma^{*} \in\left(0, \frac{1}{\sqrt{19}}\right)$ such that $h\left(\gamma^{*}\right)=0, h(\gamma)>0$ for $0<\gamma<\gamma^{*}$, and $h(\gamma)<0$ for $\gamma>\gamma^{*}$.

(iv) If $0<\gamma<\gamma^{*}$, then $-2 \pi<-\hat{\tau}_{1}(\gamma)<\hat{\tau}_{2}(\gamma)<-\frac{3 \pi}{2}$; see statement (iii). Therefore, by using that $\partial \varphi / \partial \tau>0$ in $\tau \in\left(-2 \pi,-\frac{3 \pi}{2}\right)$ (Lemma 3.1(ii)), it follows that $\varphi\left(-\hat{\tau}_{1}(\gamma), \gamma\right)<$ $\varphi\left(\hat{\tau}_{2}(\gamma), \gamma\right)=1$. The other inequality of the statement follows in a similar way.

The boundary value $\frac{1}{\sqrt{19}}$ appearing in statement (iii) of Lemma 3.2 has only computational meaning. In fact, it has been chosen to obtain the value $1 / 2$ in the statement of Theorem 1.1.

In the next result we prove the existence of a simple two-dimensional heteroclinic orbit in the piecewise linear differential system (1.5) for every value of the parameter $\lambda$ greater than a certain value $\lambda^{*}$.

Proposition 3.3. There exists a value $\lambda^{*}$ in $\left(0, \frac{1}{2}\right)$ such that

(i) if $\lambda \geq \lambda^{*}$, system (1.5) has a two-dimensional heteroclinic orbit with exactly one intersection point with the plane $\{x=0\}$, and

(ii) if $\lambda<\lambda^{*}$, system (1.5) has no two-dimensional heteroclinic orbits with exactly one intersection point with the plane $\{x=0\}$.

Proof. For the sake of simplicity, let us consider the change of variables

$$
\tau=\frac{\sqrt{4+3 \lambda^{2}}}{2} t, \quad \gamma=\frac{\lambda}{\sqrt{4+3 \lambda^{2}}}
$$

In these new variables, system (3.4) and condition (3.5) can be written as

$$
\left\{\begin{array} { l } 
{ \varphi ( - \tau , - \gamma ) = 0 , } \\
{ \varphi ( \tau , - \gamma ) \geq 1 , }
\end{array} \text { and } \left\{\begin{array}{l}
\varphi(-\bar{\tau},-\gamma)>0 \\
\text { for every } \bar{\tau} \text { in }(0, \tau)
\end{array}\right.\right.
$$

Hence, the existence of a solution $\left(\tau_{0} ; \gamma_{0}\right)$ to $(3.7)$ is equivalent to the existence of a twodimensional heteroclinic orbit with exactly one intersection point with the plane $\{x=0\}$. According to this, the proof of this proposition is reduced to the analysis of the existence of a solution to system (3.7).

Let $\gamma^{*}$ be the value defined in Lemma 3.2(iii). Since $0<\gamma^{*}<\frac{1}{\sqrt{19}}$, the corresponding value $\lambda^{*}$ given by (3.6) is real and positive and satisfies $0<\lambda^{*}<\frac{1}{2}$. Let us prove that this is the value $\lambda^{*}$ for which both statements of the proposition hold.

(i) For $\lambda_{0} \geq \lambda^{*}$, the corresponding value $\gamma_{0}$ given by (3.6) satisfies $\gamma_{0} \geq \gamma^{*}$. Thus, using Lemmas 3.1(i) and 3.2(iv), we obtain $\varphi\left(\hat{\tau}_{1}\left(\gamma_{0}\right),-\gamma_{0}\right) \geq 1$. On the other hand, from Lemma 3.1(i) and (iv), we have $\varphi\left(-\hat{\tau}_{1}\left(\gamma_{0}\right),-\gamma_{0}\right)=0$ and $\varphi\left(-\tau,-\gamma_{0}\right)>0$ for every $\tau \in\left(0, \hat{\tau}_{1}\left(\gamma_{0}\right)\right)$. That is, $\left(\hat{\tau}_{1}\left(\gamma_{0}\right) ; \gamma_{0}\right)$ is the desired solution of system (3.7).

(ii) For $\lambda_{0}<\lambda^{*}$, the corresponding value $\gamma_{0}$ given by (3.6) satisfies $\gamma_{0}<\gamma^{*}$. Note that the unique solution $\bar{\tau}$ of $\varphi\left(-\tau,-\gamma_{0}\right)=0$ which satisfies $\varphi\left(-\tau,-\gamma_{0}\right)>0$ for every $\tau \in(0, \bar{\tau})$ is $\bar{\tau}=\hat{\tau}_{1}\left(\gamma_{0}\right)$; see Lemma 3.1(i) and (iv). Because of $\gamma_{0}<\gamma^{*}$ we have $\varphi\left(\hat{\tau}_{1}\left(\gamma_{0}\right),-\gamma_{0}\right)<1$; see Lemma 3.2(iv). Consequently, we concluded that the system (3.7) does not have any solutions.

Note that a value $\lambda$ satisfying the statements of Proposition 3.3 has to be unique in $\lambda>0$. Moreover, the value $\lambda^{*} \approx 0.41527324$ can be numerically obtained. 
4. Existence of a one-dimensional heteroclinic orbit. In this section we prove the existence of a simple one-dimensional heteroclinic orbit $\rho_{\mp}$, that is, a heteroclinic orbit $\rho_{\mp}=$ $W^{u}\left(\mathbf{p}_{-}\right)=W^{s}\left(\mathbf{p}_{+}\right)$which intersects the plane $\{x=0\}$ at exactly three points. Two of these points are necessarily $\mathbf{m}_{-}$and $\mathbf{m}_{+}$.

An equivalent condition for the existence of a simple one-dimensional heteroclinic orbit is $\Pi_{+}\left(\mathbf{m}_{-}\right)=\Pi_{-}^{-1}\left(\mathbf{m}_{+}\right)$. Due to reversibility, this occurs if and only if the point $\Pi_{+}\left(\mathbf{m}_{-}\right)$ belongs to the reversibility axis, that is, when the first and third coordinates of $\Pi_{+}\left(\mathbf{m}_{-}\right)$are equal to zero. Therefore, system (1.5) has a simple one-dimensional heteroclinic orbit if and only if the system

$$
\left\{\begin{array}{l}
x_{\mathbf{m}_{-}}(t ; \lambda)=0 \\
z_{\mathbf{m}_{-}}(t ; \lambda)=0
\end{array}\right.
$$

has a solution $\left(t_{1} ; \lambda_{1}\right)$ such that $t_{1}>0, \lambda_{1}>0$, and

$$
x_{\mathbf{m}_{-}}\left(t ; \lambda_{1}\right)>0 \text { for every } t \in\left(0, t_{1}\right) \text {. }
$$

Note that condition (4.2) ensures that the point $\mathbf{x}_{\mathbf{m}_{-}}\left(t_{1} ; \lambda_{1}\right)$ is the image of $\mathbf{m}_{-}$by the Poincaré map $\Pi_{+}$.

Now, to prove the existence of solutions of system (4.1) we are going to simplify its equations. Integrating $\dot{\mathbf{x}}=A^{+} \mathbf{x}+\mathbf{e}_{3}$ in forward time with initial condition $\mathbf{x}(0 ; \lambda)=\mathbf{m}_{-}$, system (4.1) can be written as

$$
\left\{\begin{array}{c}
\frac{e^{-\lambda t}\left[\left(1+\lambda^{2}\right) \sqrt{4+3 \lambda^{2}}+2 e^{\frac{3}{2} \lambda t} \lambda^{2} \sqrt{4+3 \lambda^{2}} \cos \left(\frac{\sqrt{4+3 \lambda^{2}}}{2} t\right)-6 e^{\frac{3}{2} \lambda t} \lambda^{3} \sin \left(\frac{\sqrt{4+3 \lambda^{2}}}{2} t\right)\right]}{\lambda \sqrt{4+3 \lambda^{2}}\left(1+\lambda^{2}\right)\left(1+3 \lambda^{2}\right)}=\frac{1}{\lambda\left(1+\lambda^{2}\right)}, \\
\frac{e^{-\lambda t} \lambda\left[-\left(1+\lambda^{2}\right) \sqrt{4+3 \lambda^{2}}+2 e^{\frac{3}{2} \lambda t}\left(1+2 \lambda^{2}\right) \sqrt{4+3 \lambda^{2}} \cos \left(\frac{\sqrt{4+3 \lambda^{2}}}{2} t\right)-2 \lambda e^{\frac{3}{2} \lambda t} \sin \left(\frac{\sqrt{4+3 \lambda^{2}}}{2} t\right)\right]}{\sqrt{4+3 \lambda^{2}}\left(1+\lambda^{2}\right)\left(1+3 \lambda^{2}\right)}=0 .
\end{array}\right.
$$

Adding $\frac{\lambda\left(1+\lambda^{2}\right)}{2 e^{\frac{\lambda}{2} t}}$ times the first equation to $\frac{\left(1+\lambda^{2}\right)}{2 \lambda e^{\frac{\lambda}{2} t}}$ times the second one gives

$$
\cos \left(\frac{\sqrt{4+3 \lambda^{2}}}{2} t\right)-\frac{\lambda}{\sqrt{4+3 \lambda^{2}}} \sin \left(\frac{\sqrt{4+3 \lambda^{2}}}{2} t\right)=\frac{1}{2 e^{\frac{\lambda}{2} t}} .
$$

Moreover, multiplying the second equation by $\frac{1}{\lambda} e^{\lambda t}\left(1+\lambda^{2}\right)\left(1+3 \lambda^{2}\right)$, adding $1+\lambda^{2}$ to both sides of the obtained expression, and multiplying the result by $\frac{1}{2} e^{-\frac{3}{2} \lambda t}$, we get

$$
\left(1+2 \lambda^{2}\right) \cos \left(\frac{\sqrt{4+3 \lambda^{2}}}{2} t\right)-\frac{\lambda}{\sqrt{4+3 \lambda^{2}}} \sin \left(\frac{\sqrt{4+3 \lambda^{2}}}{2} t\right)=\frac{1+\lambda^{2}}{2 e^{\frac{3}{2} \lambda t}} .
$$

Now, the trigonometric functions are determined by solving the system given by (4.3) and $(4.4)$,

$$
\begin{aligned}
\cos \left(\frac{\sqrt{4+3 \lambda^{2}}}{2} t\right) & =\frac{1+\lambda^{2}-e^{\lambda t}}{4 \lambda^{2} e^{\frac{3}{2} \lambda t}}, \\
\sin \left(\frac{\sqrt{4+3 \lambda^{2}}}{2} t\right) & =\frac{\sqrt{4+3 \lambda^{2}}}{4 \lambda^{3} e^{\frac{3}{2} \lambda t}}\left(1+\lambda^{2}-e^{\lambda t}\left(1+2 \lambda^{2}\right)\right),
\end{aligned}
$$

Copyright (c) by SIAM. Unauthorized reproduction of this article is prohibited. 
and thus

$$
\begin{aligned}
& \cos \left(\frac{\sqrt{4+3 \lambda^{2}}}{2} t\right)^{2}+\sin \left(\frac{\sqrt{4+3 \lambda^{2}}}{2} t\right)^{2} \\
& =\frac{4 \lambda^{6}}{1+\lambda^{2}} e^{3 \lambda t}-\left(1+4 \lambda^{2}+3 \lambda^{4}\right) e^{2 \lambda t}+\left(2+6 \lambda^{2}+3 \lambda^{4}\right) e^{\lambda t}-\left(1+\lambda^{2}\right)^{2}+1
\end{aligned}
$$

or, equivalently,

$$
\frac{4 \lambda^{6}}{1+\lambda^{2}} e^{3 \lambda t}-\left(1+4 \lambda^{2}+3 \lambda^{4}\right) e^{2 \lambda t}+\left(2+6 \lambda^{2}+3 \lambda^{4}\right) e^{\lambda t}-\left(1+\lambda^{2}\right)^{2}=0 .
$$

Let us consider the system given by (4.3) and (4.6). Note that every solution of system (4.1) is a solution of system (4.3) and (4.6), but the converse is not necessarily true. In the following lemma we establish the conditions on a solution of system (4.3) and (4.6) for being a solution of system (4.1).

Lemma 4.1. Let $\left(t_{1} ; \lambda_{1}\right)$ be a solution of system (4.3) and (4.6), with $t_{1}>0$ and $\lambda_{1}>0$. Then, $\left(t_{1} ; \lambda_{1}\right)$ is a solution of system (4.1) if and only if $(2 k-1) \pi<\frac{\sqrt{4+3 \lambda_{1}^{2}}}{2} t_{1}<2 k \pi$ with $k \in \mathbb{N}$.

Proof. Suppose that $\left(t_{1} ; \lambda_{1}\right)$ is a solution of system (4.1). Since $t_{1}>0$ and $\lambda_{1}>0$, from $(4.5)$ it is clear that $\sin \left(\frac{\sqrt{4+3 \lambda_{1}^{2}}}{2} t_{1}\right)<0$. Thus, the argument $\frac{\sqrt{4+3 \lambda_{1}^{2}}}{2} t_{1}$ belongs to $((2 k-1) \pi, 2 k \pi)$ with $k \in \mathbb{N}$.

For the other implication, let us consider the system

$$
\left\{\begin{aligned}
X-\frac{\lambda_{1}}{\sqrt{4+3 \lambda_{1}^{2}}} Y & =\frac{1}{2 e^{\frac{\lambda_{1}}{2} t_{1}}}, \\
X^{2}+Y^{2} & =1
\end{aligned}\right.
$$

whose equations correspond to a straight line and a circle. Hence, it has at most two different solutions. Since the straight line defined by the first equation contains a point with $X=1$ and $Y>0$, at most one of such solutions has a negative second coordinate.

Note that

$$
(X, Y)=\left(\frac{1+\lambda_{1}^{2}-e^{\lambda_{1} t_{1}}}{4 \lambda_{1}^{2} e^{\frac{3}{2} \lambda_{1} t_{1}}}, \frac{\sqrt{4+3 \lambda_{1}^{2}}}{4 \lambda_{1}^{3} e^{\frac{3}{2} \lambda_{1} t_{1}}}\left(1+\lambda_{1}^{2}-e^{\lambda_{1} t_{1}}\left(1+2 \lambda_{1}^{2}\right)\right)\right)
$$

is a solution of system (4.7) with $Y<0$. On the other hand, since $\left(t_{1} ; \lambda_{1}\right)$ is a solution of system (4.3) and (4.6), then

$$
(\tilde{X}, \tilde{Y})=\left(\cos \left(\frac{\sqrt{4+3 \lambda_{1}^{2}}}{2} t_{1}\right), \sin \left(\frac{\sqrt{4+3 \lambda_{1}^{2}}}{2} t_{1}\right)\right)
$$

is also a solution of system (4.7) with $\tilde{Y}<0$. Therefore, we conclude that $(\tilde{X}, \tilde{Y})=(X, Y)$, which means that $\left(t_{1} ; \lambda_{1}\right)$ is a solution of (4.5) or, equivalently, a solution of (4.1).

In the next result we prove that system (4.1) has at least a solution $\left(t_{1} ; \lambda_{1}\right)$ which also satisfies some important conditions to verify inequality (4.2).

Copyright $@$ by SIAM. Unauthorized reproduction of this article is prohibited. 
Lemma 4.2. System (4.1) has a solution $\left(t_{1} ; \lambda_{1}\right)$ satisfying

$$
\frac{1}{2}<\lambda_{1}<1 \text { and } \pi<\frac{\sqrt{4+3 \lambda_{1}^{2}}}{2} t_{1}<2 \pi
$$

Proof. Let us define $\psi(s, \lambda)=\frac{4 \lambda^{6}}{1+\lambda^{2}} s^{3}-\left(1+4 \lambda^{2}+3 \lambda^{4}\right) s^{2}+\left(2+6 \lambda^{2}+3 \lambda^{4}\right) s-\left(1+\lambda^{2}\right)^{2}$ and $\tau=\frac{\sqrt{4+3 \lambda^{2}}}{2}$. Thus, system (4.3) and (4.6) can be written as

$$
\left\{\begin{array}{l}
f_{1}(\tau, \lambda)=\varphi\left(\tau, \frac{\lambda}{\sqrt{4+3 \lambda^{2}}}\right)-\frac{1}{2}=0 \\
f_{2}(\tau, \lambda)=\psi\left(e^{\frac{2 \lambda}{\sqrt{4+3 \lambda^{2}}} \tau}, \lambda\right)=0
\end{array}\right.
$$

where $\varphi$ is the function defined in Lemma 3.1.

From Lemma 4.1, the proof is complete by showing the existence of a solution $\left(\tau_{1} ; \lambda_{1}\right)$ of system $(4.8)$ in $(\pi, 2 \pi) \times\left(\frac{1}{2}, 1\right)$. We will obtain such a solution by applying the Poincaré Miranda theorem [17], which is an $n$-dimensional extension of Bolzano's theorem.

The definition of $\varphi$ makes it obvious that $f_{1}(\pi, \lambda)>0$ and $f_{1}(2 \pi, \lambda)<0$ for every $\lambda>0$. In particular, it is true for $\lambda \in\left[\frac{1}{2}, 1\right]$. That is, function $f_{1}$ takes different signs at the vertical sides of the rectangle $[\pi, 2 \pi] \times\left[\frac{1}{2}, 1\right]$.

Let us now analyze the sign of function $f_{2}(\tau, \lambda)$ at the horizontal sides of $[\pi, 2 \pi] \times\left[\frac{1}{2}, 1\right]$ by studying the cubic polynomials $\psi(s, 1)$ and $\psi\left(s, \frac{1}{2}\right)$. Since the derivative of $\psi(s, 1)$ with respect to $s$ is positive in $\mathbb{R}$ and $\psi(1,1)=1$, we have $\psi(s, 1)>1$ for every $s \in(1,+\infty)$. Therefore, $f_{2}(\tau, 1)>1$ for every $\tau>0$ and, in particular, $f_{2}(\tau, 1)>0$ for every $\tau \in[\pi, 2 \pi]$.

For the last side of the rectangle, straightforward computations show that the derivative of $\psi\left(s, \frac{1}{2}\right)$ with respect to $s$ vanishes at two values $s_{1}<1<s_{2}$, where $s_{2}$ is a local minimum. Taking into account that $\psi\left(1, \frac{1}{2}\right)=-\frac{1}{80}$ and $\psi\left(27, \frac{1}{2}\right)=-\frac{41003}{80}$, it follows that $\psi\left(s, \frac{1}{2}\right)<0$ for $s \in[1,27]$. Note that, for $\lambda=\frac{1}{2}$ and $\tau \in[\pi, 2 \pi]$, the exponential function $e^{\frac{2 \lambda}{\sqrt{4+3 \lambda^{2}}} \tau}$ takes values in $[1,27]$, as is easy to check from inequalities $4 \pi<3 \sqrt{19}$ and $e<3$. Therefore, $f_{2}\left(\tau, \frac{1}{2}\right)<0$ for every $\tau \in[\pi, 2 \pi]$.

The lemma follows by the Poincaré-Miranda theorem.

To conclude the proof of the existence of a simple one-dimensional heteroclinic orbit for system (1.5), it remains only to check that the solution $\left(t_{1} ; \lambda_{1}\right)$ of system (4.1), given in Lemma 4.2, also satisfies condition (4.2). This is done in the following result.

Proposition 4.3. For $\lambda=\lambda_{1}$, the system (1.5) has a simple one-dimensional heteroclinic orbit.

Proof. From Lemma 4.2, there exists a solution $\left(t_{1} ; \lambda_{1}\right)$ of system (4.1). Integrating $\dot{\mathbf{x}}=A^{+} \mathbf{x}+\mathbf{e}_{3}$ in forward time with initial condition $\mathbf{x}(0 ; \lambda)=\mathbf{m}_{-}$, we obtain the following expression for the second coordinate of the solution:

$$
y_{\mathbf{m}_{-}}(t ; \lambda)=\frac{2 e^{\frac{1}{2} \lambda t} \lambda^{2} \sqrt{4+3 \lambda^{2}} \cos \left(\frac{\sqrt{4+3 \lambda^{2}}}{2} t\right)+2 e^{\frac{1}{2} \lambda t} \lambda\left(2+3 \lambda^{2}\right) \sin \left(\frac{\sqrt{4+3 \lambda^{2}}}{2} t\right)}{\sqrt{4+3 \lambda^{2}}\left(1+\lambda^{2}\right)\left(1+3 \lambda^{2}\right)}+\frac{e^{-\lambda t}}{\left(1+3 \lambda^{2}\right)}
$$




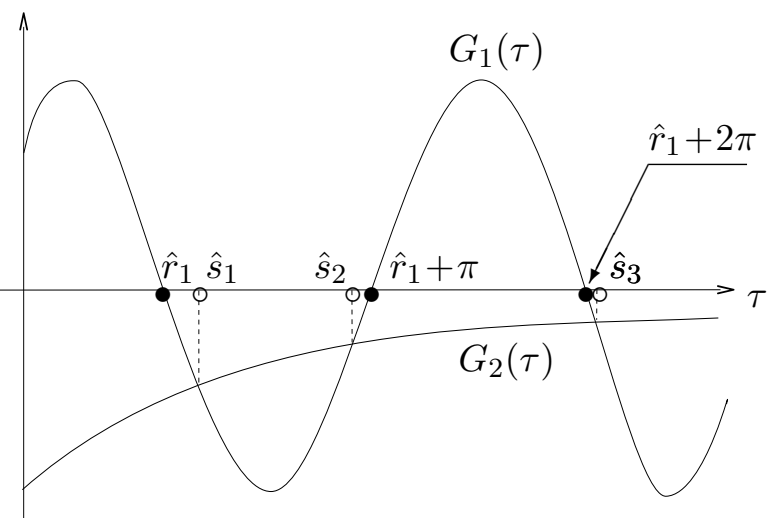

Figure 3. Qualitative behavior of functions $G_{1}$ and $G_{2}$.

Remember that, in our system, $\dot{x}_{\mathbf{m}_{-}}(t ; \lambda)=y_{\mathbf{m}_{-}}(t ; \lambda)$. Thus, using $(4.5)$ to simplify $y_{\mathbf{m}_{-}}\left(t_{1} ; \lambda_{1}\right)$, we obtain

$$
\dot{x}_{\mathbf{m}_{-}}\left(t_{1} ; \lambda_{1}\right)=\frac{e^{-\lambda_{1} t_{1}}-1}{\lambda_{1}^{2}}<0 .
$$

On the other side, $\dot{x}_{\mathbf{m}_{-}}\left(0 ; \lambda_{1}\right)>0$.

Assume that $\left(t_{1} ; \lambda_{1}\right)$ does not satisfy condition (4.2). Therefore, there exists a value in $\left(0, t_{1}\right)$ where $x_{\mathbf{m}_{-}}\left(t ; \lambda_{1}\right)$ vanishes. From $\dot{x}_{\mathbf{m}_{-}}\left(0 ; \lambda_{1}\right)>0$ and $\dot{x}_{\mathbf{m}_{-}}\left(t_{1} ; \lambda_{1}\right)<0$ it may be concluded that the derivative $\dot{x}_{\mathbf{m}_{-}}\left(t ; \lambda_{1}\right)$, that is, $y_{\mathbf{m}_{-}}\left(t ; \lambda_{1}\right)$, has to vanish at least at three values $s_{1}, s_{2}, s_{3}$ such that $0<s_{1}<s_{2}<s_{3}<t_{1}$. For simplicity of notation, we consider the change $\tau=\frac{\sqrt{4+3 \lambda_{1}^{2}}}{2} t$. Let $\hat{s}_{1}, \hat{s}_{2}, \hat{s}_{3}, \tau_{1}$ denote the respective values of $s_{1}, s_{2}, s_{3}, t_{1}$ by this change. Therefore, $0<\hat{s}_{1}<\hat{s}_{2}<\hat{s}_{3}<\tau_{1}$.

From (4.9), the equality $y_{\mathbf{m}_{-}}\left(t ; \lambda_{1}\right)=0$ is equivalent to $G_{1}(\tau)=G_{2}(\tau)$, where

$$
\begin{aligned}
& G_{1}(\tau)=\frac{\lambda_{1}^{2}}{1+\lambda_{1}^{2}} \cos (\tau)+\frac{\lambda_{1}\left(2+3 \lambda_{1}^{2}\right)}{\sqrt{4+3 \lambda_{1}^{2}}\left(1+\lambda_{1}^{2}\right)} \sin (\tau), \\
& G_{2}(\tau)=-\frac{1}{2} \exp \left(\frac{-3 \lambda_{1}}{\sqrt{4+3 \lambda_{1}^{2}}} \tau\right) .
\end{aligned}
$$

The qualitative behavior of $G_{1}$ and $G_{2}$ is shown in Figure 3.

Since $G_{2}(\tau)<0$ for every $\tau$ and $G_{1}(0)>0$, there exists an $\hat{r}_{1} \in\left(0, \hat{s}_{1}\right)$ where $G_{1}\left(\hat{r}_{1}\right)=0$, $G_{1}(\tau)<0$ for $\tau \in \bigcup_{k=0}^{\infty} I_{k}$, where $I_{k}=\left(\hat{r}_{1}+2 k \pi, \hat{r}_{1}+(2 k+1) \pi\right)$. On the other hand, the second derivative $G_{2}^{\prime \prime}$ is always negative while the second derivative $G_{1}^{\prime \prime}$ is positive in every interval $I_{k}$ with $k=0,1,2, \ldots$ Therefore, in each interval $I_{k}$ there may exist at most two values where $G_{1}$ and $G_{2}$ coincide.

We conclude that $\hat{s}_{3}>\hat{r}_{1}+2 \pi$. In particular, $\tau_{1}>2 \pi$ and, equivalently, $\frac{\sqrt{4+3 \lambda_{1}^{2}}}{2} t_{1}>2 \pi$, which contradicts Lemma 4.2. Thus, the proposition follows.

From Propositions 3.3 and 4.3 we conclude that for $\lambda=\lambda_{1}$ system (1.5) has a simple twodimensional heteroclinic orbit $\rho_{ \pm}$and a simple one-dimensional heteroclinic orbit $\rho_{\mp}$. That is, for $\lambda=\lambda_{1}$ system (1.5) has a $(3,1)$ heteroclinic cycle $\Gamma=\mathbf{p}_{+} \cup \rho_{ \pm} \cup \mathbf{p}_{-} \cup \rho_{\mp}$. 
5. Existence of a reversible T-point heteroclinic cycle. In this section we finish the proof of Theorem 1.1. It remains only to verify that the heteroclinic cycle $\Gamma$, obtained in section 4 for $\lambda=\lambda_{1}$, satisfies the two following conditions, which are equivalent to those stated at the definition of a reversible T-point heteroclinic cycle:

(i) Manifolds $W^{u}\left(\mathbf{p}_{+}\right)$and $W^{s}\left(\mathbf{p}_{-}\right)$intersect transversally along the two-dimensional heteroclinic orbit $\rho_{ \pm}$.

(ii) The difference between the third coordinates of $\Pi_{+}\left(\mathbf{m}_{-}\right)$and $\Pi_{-}^{-1}\left(\mathbf{m}_{+}\right)$is diffeomorphic to $\mu=\lambda-\lambda_{1}$ (note that the distance between two points in the plane $\{x=0\}$ which are symmetric with respect to $\mathbf{R}$ is two times the absolute value of the third coordinate of any of them).

Statement (i) is a direct consequence of the transversality of manifolds $W^{u}\left(\mathbf{p}_{+}\right)$and $W^{s}\left(\mathbf{p}_{-}\right)$at $\{x=0\}$ and the differentiability of the flow.

To verify condition (ii), it is necessary only to prove that the third coordinate of $\Pi_{+}\left(\mathbf{m}_{-}\right)$ is diffeomorphic to $\mu=\lambda-\lambda_{1}$.

Let $t_{\mathbf{m}_{-}}^{+}(\lambda)$ be the flying time of the solution through the point $\mathbf{m}_{-}$for each $\lambda$ in a neighborhood of $\lambda_{1}$. In particular, $t_{\mathbf{m}_{-}}^{+}\left(\lambda_{1}\right)=t_{1}$, where $t_{1}$ is defined in Lemma 4.2. The third coordinate of $\Pi_{+}\left(\mathbf{m}_{-}\right)$, as a function of $\lambda$, can be written as $h(\lambda)=z_{\mathbf{m}_{-}}\left(t_{\mathbf{m}_{-}}^{+}(\lambda) ; \lambda\right)$. Notice that $h(\lambda)$ is analytical because the solution depends only on the linear system in the half-space $\{x>0\}$ and the orbit through $\Pi_{+}\left(\mathbf{m}_{-}\right)$is transversal to the plane $\{x=0\}$ for $\lambda=\lambda_{1}$. Thus, since $h\left(\lambda_{1}\right)=0$, to conclude that $h(\lambda)$ is diffeomorphic to $\mu=\lambda-\lambda_{1}$ it is sufficient to prove that $h^{\prime}\left(\lambda_{1}\right) \neq 0$.

Proposition 5.1. Function $h(\lambda)=z_{\mathbf{m}_{-}}\left(t_{\mathbf{m}_{-}}^{+}(\lambda) ; \lambda\right)$ satisfies $h^{\prime}\left(\lambda_{1}\right)<0$.

Proof. The first derivative $h^{\prime}\left(\lambda_{1}\right)$ is given by

$$
\left.\frac{d h}{d \lambda}\right|_{\lambda_{1}}=\left.\dot{z}_{\mathbf{m}_{-}}\left(t_{1} ; \lambda_{1}\right) \frac{d t_{\mathbf{m}_{-}}^{+}}{d \lambda}\right|_{\lambda_{1}}+\left.\frac{\partial z_{\mathbf{m}_{-}}(t ; \lambda)}{\partial \lambda}\right|_{\left(t_{1} ; \lambda_{1}\right)} .
$$

Since the derivative $\dot{x}_{\mathbf{m}_{-}}\left(t_{1} ; \lambda_{1}\right)=y_{\mathbf{m}_{-}}\left(t_{1} ; \lambda_{1}\right)$ does not vanish, the function $t_{\mathbf{m}_{-}}^{+}(\lambda)$ is implicitly defined by the equation $x_{\mathbf{m}_{-}}(t ; \lambda)=0$ in a neighborhood of $\left(t_{1} ; \lambda_{1}\right)$. Moreover, $t_{\mathbf{m}_{-}}^{+}(\lambda)$ is analytical and

$$
\left.\frac{d t_{\mathbf{m}_{-}}^{+}}{d \lambda}\right|_{\lambda_{1}}=-\frac{\left.\frac{\partial x_{\mathbf{m}_{-}}(t ; \lambda)}{\partial \lambda}\right|_{\left(t_{1} ; \lambda_{1}\right)}}{y_{\mathbf{m}_{-}}\left(t_{1} ; \lambda_{1}\right)} .
$$

On the other hand, since $z_{\mathbf{m}_{-}}(t ; \lambda)$ is the third coordinate of the solution of system $(1.5)$ with initial condition $\mathbf{m}_{-}$, we have

$$
\dot{z}_{\mathbf{m}_{-}}\left(t_{1} ; \lambda_{1}\right)=-\lambda_{1}\left(1+\lambda_{1}^{2}\right) x_{\mathbf{m}_{-}}\left(t_{1} ; \lambda_{1}\right)-y_{\mathbf{m}_{-}}\left(t_{1} ; \lambda_{1}\right)+1=-y_{\mathbf{m}_{-}}\left(t_{1} ; \lambda_{1}\right)+1 .
$$

Therefore, substituting into the expression of $d h / d \lambda$, we obtain

$$
\left.\frac{d h}{d \lambda}\right|_{\lambda_{1}}=\left.\frac{y_{\mathbf{m}_{-}}\left(t_{1} ; \lambda_{1}\right)-1}{y_{\mathbf{m}_{-}}\left(t_{1} ; \lambda_{1}\right)} \frac{\partial x_{\mathbf{m}_{-}}(t ; \lambda)}{\partial \lambda}\right|_{\left(t_{1} ; \lambda_{1}\right)}+\left.\frac{\partial z_{\mathbf{m}_{-}}(t ; \lambda)}{\partial \lambda}\right|_{\left(t_{1} ; \lambda_{1}\right)} .
$$


Integrating the linear system $\dot{\mathbf{x}}=A^{+} \mathbf{x}+\mathbf{e}_{3}$ with initial condition $\mathbf{m}_{-}$and taking into account that $x_{\mathbf{m}_{-}}\left(t_{1} ; \lambda_{1}\right)=0$ and $z_{\mathbf{m}_{-}}\left(t_{1} ; \lambda_{1}\right)=0$, the following equalities hold:

$$
\begin{aligned}
y_{\mathbf{m}_{-}}\left(t_{1} ; \lambda_{1}\right) & =\frac{e^{-\lambda_{1} t_{1}}-1}{\lambda_{1}^{2}}, \\
\left.\frac{\partial x_{\mathbf{m}_{-}}(t ; \lambda)}{\partial \lambda}\right|_{\left(t_{1} ; \lambda_{1}\right)} & =\frac{\lambda_{1} t_{1}}{\lambda_{1}^{2}\left(1+3 \lambda_{1}^{2}\right)\left(1+\lambda^{2}\right)\left(4+3 \lambda^{2}\right)}\left(9 \lambda_{1}^{4}\left(e^{-\lambda_{1} t_{1}}-1\right)+18 \lambda^{2}\left(e^{-\lambda_{1} t_{1}}-1\right)\right. \\
& \left.+9 e^{-\lambda_{1} t_{1}}-5\right) \\
& +\frac{2}{\lambda_{1}^{2}\left(1+3 \lambda_{1}^{2}\right)\left(1+\lambda^{2}\right)\left(4+3 \lambda^{2}\right)}\left(3 \lambda_{1}^{2}\left(2 e^{-\lambda_{1} t_{1}}-3\right)+7\left(e^{-\lambda_{1} t_{1}}-1\right)\right), \\
& =\frac{3 \lambda_{1} t_{1}}{\left.\lambda_{\mathbf{m}_{-}}^{2}(t ; \lambda)\right|_{\left(t_{1} ; \lambda_{1}\right)}\left(1+3 \lambda_{1}^{2}\right)\left(1+\lambda^{2}\right)\left(4+3 \lambda^{2}\right)}\left(3 \lambda_{1}^{6}+\lambda_{1}^{4}\left(7-e^{-\lambda_{1} t_{1}}\right)+\lambda_{1}^{2}\left(5-2 e^{-\lambda_{1} t_{1}}\right)\right. \\
+\frac{2}{\lambda_{1}^{2}\left(1+3 \lambda_{1}^{2}\right)\left(1+\lambda^{2}\right)\left(4+3 \lambda^{2}\right)}\left(3 \lambda_{1}^{4}\left(e^{-\lambda_{1} t_{1}}-1\right)+\lambda_{1}^{2}\left(3 e^{-\lambda_{1} t_{1}}-2\right)\right. & \left.+1-e^{-\lambda_{1} t_{1}}\right) .
\end{aligned}
$$

Straightforward computations show that

$$
\left.\frac{d h}{d \lambda}\right|_{\lambda_{1}}=-\frac{2}{\lambda_{1}^{2}\left(1+3 \lambda_{1}^{2}\right)\left(1+\lambda^{2}\right)\left(4+3 \lambda^{2}\right)\left(e^{\lambda_{1} t_{1}}-1\right)} P\left(t_{1}, \lambda_{1}\right),
$$

where

$$
\begin{aligned}
P\left(t_{1}, \lambda_{1}\right) & =3\left(e^{\lambda_{1} t_{1}}+e^{-\lambda_{1} t_{1}}-2\right) \lambda_{1}^{5} t_{1}+3\left(4 e^{\lambda_{1} t_{1}}+e^{-\lambda_{1} t^{*}}-4\right) \lambda_{1}^{4}+2\left(2 e^{\lambda_{1} t_{1}}+3 e^{-\lambda_{1} t_{1}}-6\right) \lambda^{3} t_{1} \\
& +9\left(2 e^{\lambda_{1} t_{1}}+e^{-\lambda_{1} t_{1}}-3\right) \lambda_{1}^{2}+\left(e^{\lambda t_{1}}+3 e^{-\lambda_{1} t_{1}}-4 \lambda_{1} t_{1}\right)+6\left(e^{\lambda_{1} t_{1}}+e^{-\lambda_{1} t_{1}}-2\right) .
\end{aligned}
$$

From Lemma 4.2 we have $\frac{1}{2}<\lambda_{1}<1$ and $\frac{2 \pi}{\sqrt{7}}<t_{1}<\frac{8 \pi}{\sqrt{19}}$. Therefore, $\frac{\pi}{\sqrt{7}}<\lambda_{1} t_{1}<\frac{8 \pi}{\sqrt{19}}$ and $3<e^{\lambda_{1} t_{1}}$. This implies that the coefficients of $\lambda^{5}, \lambda^{4}, \lambda^{3}$, and $\lambda^{2}$ in the expression of $P\left(\lambda_{1}, t_{1}\right)$ are positive. Moreover, $e^{\lambda t_{1}}+3 e^{-\lambda_{1} t_{1}}-4 \lambda_{1} t_{1}>-\lambda_{1} t_{1}>-\frac{8 \pi}{\sqrt{19}}$, and $6\left(e^{\lambda_{1} t_{1}}+e^{-\lambda_{1} t_{1}}-2\right)>6$. Since $6 \sqrt{19}>8 \pi$, it follows that $P\left(\lambda_{1}, t_{1}\right)>0$, which proves the proposition.

From Proposition 5.1 we finish the proof of Theorem 1.1.

Acknowledgments. The authors would like to thank Dr. E. Freire, Dr. E. Ponce, and Dr. F. Torres for useful discussions.

\section{REFERENCES}

[1] A. A. Andronov, A. A. Vitt, And S. Khaikin, Theory of Oscillators, Dover, New York, 1987.

[2] A. Arneodo, P. Coullet, And C. Tresser, Possible new strange attractors with spiral structure, Comm. Math. Phys., 79 (1981), pp. 573-579.

[3] V. V. Bykov, The bifurcations of separatrix contours and chaos, Phys. D, 62 (1993), pp. 290-299. 
[4] V. V. Bykov, On systems with separatrix contour containing two saddle-foci, J. Math. Sci. (New York), 95 (1999), pp. 2513-2522.

[5] V. V. BYKov, Orbit structure in a neighborhood of a separatrix cycle containing two saddle-foci, in Amer. Math. Soc. Transl. Ser. 2 200, AMS, Providence, RI, 2000, pp. 87-89.

[6] V. Carmona, E. Freire, E. Ponce, And F. Torres, On simplifying and classifying piecewise linear systems, IEEE Trans. Circuits Systems I Fund. Theory Appl., 49 (2002), pp. 609-620.

[7] V. Carmona, E. Freire, E. Ponce, And F. Torres, Invariant manifolds of periodic orbits for piecewise linear three-dimensional systems, IMA J. Appl. Math., 69 (2004), pp. 71-91.

[8] L. O. Chua, M. Komuro, and T. Matsumoto, The double scroll family. II. Rigorous analysis of bifurcation phenomena, IEEE Trans. Circuits and Systems, 33 (1986), pp. 1097-1118.

[9] P. Coullet, C. Tresser, And A. Arneodo, Transition to stochasticity for a class of forced oscillators, Phys. Lett. A, 72 (1979), pp. 268-270.

[10] F. Dumortier, S. IBAÑez, AND H. KoKubu, New aspects in the unfolding of the nilpotent singularity of codimension three, Dyn. Syst., 16 (2001), pp. 63-95.

[11] F. Dumortier, S. Ibañez, and H. Kokubu, Cocoon bifurcation in three-dimensional reversible vector fields, Nonlinearity, 19 (2006), pp. 305-328.

[12] F. Fernández-SÁnchez, E. Freire, And A. J. Rodríguez-Luis, T-points in a $\mathbb{Z}_{2}$-symmetric electronic oscillator. (I) Analysis, Nonlinear Dynam., 28 (2002), pp. 53-69.

[13] E. Freire, E. Gamero, A. J. Rodriguez-Luis, and A. AlgabA, A note on the triple-zero linear degeneracy: Normal forms, dynamical and bifurcation behaviors of an unfolding, Internat. J. Bifur. Chaos Appl. Sci. Engrg., 12 (2002), pp. 2799-2820.

[14] P. Glendinning And C. Sparrow, T-points: A codimension two heteroclinic bifurcation, J. Statist. Phys., 43 (1986), pp. 479-488.

[15] S. V. Gonchenko, D. V. Turaev, P. Gaspard, and G. Nicolis, Complexity in the bifurcation structure of homoclinic loops to a saddle-focus, Nonlinearity, 10 (1997), pp. 409-423.

[16] S. IBAÑEZ AND J. A. RodRIGUEZ, Shil'nikov configurations in any generic unfolding of the nilpotent singularity of codimension three on $\mathbb{R}^{3}$, J. Differential Equations, 208 (2005), pp. 147-175.

[17] W. Kulpa, The Poincaré-Miranda theorem, Amer. Math. Monthly, 104 (1997), pp. 545-550.

[18] Y. Kuramoto And T. Tsuzuki, Persistent propagation of concentration waves in dissipative media far for thermal equilibrium, Progr. Theoret. Phys., 55 (1976), pp. 356-369.

[19] J. S. W. LAmb, M. A. TexeIRA, And K. N. Webster, Heteroclinic bifurcations near Hopf-zero bifurcation in reversible vector fields in $\mathbb{R}^{3}$, J. Differential Equations, 219 (2005), pp. 78-115.

[20] Y. T. LAU, The "cocoon" bifurcation in three-dimensional systems with two fixed points, Internat. J. Bifur. Chaos Appl. Sci. Engrg., 2 (1992), pp. 543-558.

[21] J. LlibRe AND A. E. TeRuel, Existence of Poincaré maps in piecewise linear differential systems in $\mathbb{R}^{n}$, Internat. J. Bifur. Chaos Appl. Sci. Engrg., 8 (2004), pp. 2843-2851.

[22] J. Llibre, E. Ponce, And A. E. Teruel, Horseshoes near homoclinic orbits for piecewise linear differential systems in $\mathbb{R}^{3}$, Internat. J. Bifur. Chaos Appl. Sci. Engrg., 17 (2007), pp. 1171-1184.

[23] T. Matsumoto, L. O. Chua, And M. Komuro, The double scroll, IEEE Trans. Circuits and Systems, 32 (1985), pp. 797-818.

[24] T. Matsumoto, L. O. Chua, And K. Ayaki, Reality of chaos in the double scroll circuit: A computerassisted proof, IEEE Trans. Circuits and Systems, 35 (1988), pp. 908-925.

[25] D. Michelson, Steady solutions of the Kuramoto-Sivashinsky equation, Phys. D, 19 (1986), pp. 89-111.

[26] L. P. Shil'nikov, A case of the existence of a denumerable set of periodic motions, Sov. Math. Dokl., 6 (1965), pp. 163-166.

[27] L. P. ShiL'Nikov, A contribution to the problem of the structure of an extended neighbourhood of a rough equilibrium state of saddle-focus type, Math. USSR Sbornik, 10 (1970), pp. 91-102.

[28] K. N. Webster And J. N. Elgin, Asymptotic analysis of the Michelson system, Nonlinearity, 16 (2003), pp. 2149-2162.

Copyright (C) by SIAM. Unauthorized reproduction of this article is prohibited. 